\title{
Seleção da matriz de variância-covariância residual na análise de ensaios varietais com medidas repetidas em cana-de-açúcar
}

\author{
Selection of the residual variance-covariance matrix in the analysis of varietal trials with repeated \\ measures in sugarcane
}

\author{
Emerson Noleto Silva ${ }^{I}$ João Batista Duarte ${ }^{\text {II }}$ Américo José dos Santos Reis ${ }^{\text {II }}$
}

\section{RESUMO}

Este trabalho objetivou avaliar diferentes estruturas da matriz de variâncias e covariâncias residual ( $\Sigma$ ), quanto ao ajustamento de dados longitudinais via modelos mistos, em experimentos varietais de cana-de-açúcar. A seleção adequada desta matriz garante a escolha de um modelo mais representativo dos dados. Em cada modelagem, variou-se ainda a suposição associada aos efeitos de tratamentos (variedades), como fixos e aleatórios. Quatro ensaios varietais, conduzidos entre 2005 e 2009, em três localidades do Estado de Goiás, foram considerados. Cada experimento foi delineado em blocos casualizados com três ou quatro repetições. A variável resposta analisada foi toneladas de colmos por hectare (TCH). Para avaliar a qualidade de ajustamento dos modelos, foram utilizados o critério de informação de Akaike (AIC) e o teste da razão de verossimilhanças. Este último foi utilizado apenas para comparar modelos hierárquicos, tomados dois a dois. Constatou-se que as análises pelo modelo univariado clássico de parcelas subdivididas oscilaram entre as piores ou entre aquelas de ajustes apenas medianos. As estruturas da matriz $\sum$ com os melhores ajustamentos variaram entre os ensaios, com destaque para a matriz não-estruturada. Tais resultados revelam que a estrutura de erros independentes, em geral, não se mostra adequada para esse tipo de análise e, também, que não é seguro definir previamente uma estrutura especifica de $\Sigma$ para tais ensaios. Pequenas alterações foram observadas na classificação das estruturas ao se assumirem os efeitos de tratamentos como fixos ou aleatórios; porém, sem efeito importante na classificação das melhores estruturas em cada ensaio.

Palavras-chave: Saccharum spp., dados longitudinais, estruturas de covariância, modelos mistos, genótipos aleatórios.

\section{ABSTRACT}

This study aimed to evaluate different residual structures of variance-covariance matrix $(\Sigma)$, regarding the fitting of longitudinal data via mixed models in variety trials of sugarcane. The adequate choice of this matrix provides most representative models to the data. In each model was also evaluated the effects of treatments (varieties), either as fixed or as random. Four trials were carried out in three locations in the Goiás State, Brazil, from 2005 to 2009. Each experiment was designed in randomized complete block with three or four repetitions. The response variable analyzed was tons of stalks per hectare (TCH). The goodness of fitting of the different models to the data was assessed by Akaike information criterion (AIC) and by likelihood ratio test (LRT). This last statistic was used only to compare nested models, two by two. It was observed that classic model in split-plot design ranged among the worst or with just median adjustments. The structures of $\Sigma$ matrix with the best fittings to the data varied among trials, with outstanding for the unstructured matrix. These results show that the structure of independent errors, in general, is not adequate for these analyses, and a prior definition of the co-variance structure can lead to unreliable results for these trials. Small changes were observed in the ranking of these structures by assuming the treatment effects as fixed or random, however, without significant effects on the ranking of the best structures in each trial.

Key words: Saccharum spp., longitudinal data, covariance structures, mixed models, random genotypes.

\section{INTRODUÇÃO}

A avaliação de clones experimentais em cana-de-açúcar comumente envolve a amostragem na mesma planta ou parcela ao longo dos cortes (cana-planta, cana-soca, ressoca etc). Esse fato é de grande importância para avaliar as relações entre os diferentes cortes durante o desenvolvimento

IPrograma de Pós-graduação em Agronomia (PPGA), Universidade Federal de Goiás (UFG), Goiânia, GO, Brasil.

IISetor de Melhoramento de Plantas, Escola de Agronomia, UFG, CP 131, 74001-970, Goiânia, GO, Brasil. E-mail: jbduarte@ufg.br. Autor para correspondência.

IIIRede Interuniversitária para o Desenvolvimento do Setor Sucroenergético (Ridesa Brasil), Programa de Melhoramento Genético da Cana-de-Açúcar (PMGCA), UFG, Goiânia, GO, Brasil. 
dos clones. Nesse tipo de experimento, ocorre interdependência entre as observações, devido às medidas repetidas nos diferentes tempos sobre a mesma parcela, o que dificulta a análise estatística dos dados. Para contornar este problema, podemse analisar as observações (dados longitudinais) através de diferentes estruturas da matriz de variâncias-covariâncias residuais $(\Sigma)$. Porém, a apresentação adequada das observações depende de seu ajustamento ao modelo de estrutura selecionado (FREITAS et al., 2008).

Na busca do modelo estatístico que melhor represente os dados, levando em consideração se estes são independentes ou não, várias estruturas de matriz de variâncias-covariâncias $(\Sigma)$ precisam ser avaliadas (QUINTAL, 2013). Para esta finalidade, uma alternativa é utilizar a abordagem de modelos mistos, que possibilita contemplar a estrutura de interdependência das observações, acomodando aquela mais apropriada à correlação presente nas medidas repetidas, tomadas na mesma unidade experimental.

Várias técnicas de seleção do modelo com a respectiva estrutura da matriz de covariâncias podem ser utilizadas. As de uso mais difundido são os critérios de informação de Akaike - AIC (Akaike's Information Criterion) e o de Schwarz - BIC (Bayesian Information Criterion); ambos baseados na verossimilhança de ajuste do modelo e dependentes do número de observações e parâmetros (FLORIANO et al., 2006). Em geral, os dois critérios produzem resultados concordantes. Outro procedimento de uso comum é o teste assintótico de razão de verossimilhanças (LRT), que permite comparar dois modelos de cada vez, ambos ajustados por verossimilhança, um deles como versão restrita do outro (modelos aninhados ou encaixados). O uso dessas técnicas é fundamental na teoria de decisão em modelo misto, pois, além da qualidade de ajustamento, consideram o princípio da parcimônia, que penaliza modelos com maior número de parâmetros (CAMARINHA FILHO, 2002).

Nesse contexto, o objetivo deste estudo foi comparar modelos estatísticos com diferentes estruturas de matriz de variâncias-covariâncias $(\Sigma)$ para a análise de dados com medidas repetidas em experimentos varietais de cana-de-açúcar. Buscouse também avaliar a influência da suposição associada aos efeitos de tratamentos genéticos (variedades), como fixos ou aleatórios, sobre os resultados dos ajustes obtidos sob diferentes estruturas de $\Sigma$.

\section{MATERIAL E MÉTODOS}

O material de pesquisa consistiu num grupo de quatro experimentos varietais de canade-açúcar, conduzidos entre 2004 e 2009, em três localidades do Estado de Goiás: Goiatuba (usina Goiasa, com dois ensaios), Goianésia (usina Jalles Machado) e Jandaia (usina Denusa). Os ensaios foram instalados no delineamento de blocos completos casualizados, com quatro (Goiatuba e Jandaia) ou três repetições (Goianésia). Os clones ou tratamentos foram provenientes do Programa de Melhoramento Genético da Cana-de-açúcar da Universidade Federal de Goiás (PMGCAUFG), vinculado à Rede Interuniversitária para o Desenvolvimento do Setor Sucroenergético (Ridesa Brasil). Originaram-se de cruzamentos realizados em 1995 e 1996, razão por que são referidos como clones das Séries 95 e 96.

As parcelas foram constituídas de cinco fileiras de plantas, de $10 \mathrm{~m}$, com espaçamento de $1,5 \mathrm{~m}$ entre fileiras. No plantio, o número de gemas por metro de sulco variou entre oito a vinte. Neste estudo, foram considerados apenas os dados da variável tonelada de cana-de-açúcar por hectare $(\mathrm{TCH})$, nos diferentes cortes (quatro nos ensaios de Goiatuba e Goianésia, e três no ensaio de Jandaia). Em cada experimento, as avaliações foram realizadas sobre a mesma parcela, caracterizando o delineamento como "parcelas subdivididas no tempo" ou "ensaio de medidas repetidas".

A análise dos dados via abordagem de modelos mistos teve como objetivo avaliar estruturas da matriz de variâncias-covariâncias residuais diferentes de $\Sigma=\mathrm{I} \sigma^{2}$ (modelo clássico, de erros independentes), quanto à sua adequação aos dados. Assim, conforme descreve FREITAS (2007), procedeu-se à análise mediante ajustamento do seguinte modelo:

$y_{i}=X_{i} \beta+Z_{i} \gamma_{i}+e_{i}$

em que: $y_{i}$ é o vetor coluna $\left(n_{i} \times 1\right)$ das $n_{i}$ observações tomadas da unidade experimental "i" ao longo do tempo ou condição de avaliação; $\beta$ é o vetor ( $\mathrm{p}$ x 1) de parâmetros fixos desconhecidos, em que a dimensão 'p' é o número de níveis de efeitos fixos; $\mathrm{X}_{\mathrm{i}}$ é a matriz $\left(\mathrm{n}_{\mathrm{i}} \mathrm{x}\right.$ p) de incidências associadas aos elementos do vetor $\beta$; $\gamma_{\mathrm{i}}$ é o vetor ( $\left.\mathrm{g} \times 1\right)$ de efeitos aleatórios desconhecidos, assumindo-se $\gamma_{\mathrm{i}} \sim \mathrm{N}\left(0, \mathrm{G}_{\mathrm{i}}=\mathrm{I}^{2}{ }_{\mathrm{g}}\right)$, em que $\sigma_{\mathrm{g}}{ }^{2}$ é variância genotípica comum (este efeito apenas esteve presente no modelo quando se assumiu genótipos de efeitos aleatórios); $Z_{\mathrm{i}}$ é a matriz $\left(\mathrm{n}_{\mathrm{i}} \times \mathrm{g}\right)$ de incidências associadas aos elementos do vetor $\gamma_{i}$ (idem observação para $\gamma_{\mathrm{i}}$, com respeito à suposição 
de aleatoriedade para tais efeitos); $\mathrm{e}_{\mathrm{i}}$ é o vetor $\left(\mathrm{n}_{\mathrm{i}} \times 1\right)$ de erros aleatórios, com $\mathrm{e}_{\mathrm{i}} \sim \mathrm{N}\left(0, \mathrm{R}_{\mathrm{i}}=\Sigma\right)$, em que $\Sigma$ é a matriz de variâncias-covariâncias residuais, que, neste caso, assumiu diferentes estruturas.

As estruturas de matriz de variânciascovariâncias residuais $(\mathrm{R}=\Sigma)$ avaliadas, em conformidade com aplicações usuais na literatura de análise de medidas repetidas (FREITAS, 2007; QUINTAL, 2013) foram: parcela subdividida clássica (PSC) - estrutura de erros independentes baseada no ajuste do modelo univariado clássico; componente de variância (VC) - variâncias desiguais entre cortes $\left(\sigma_{i}^{2} ; i=1,2, \ldots, c\right.$ cortes $)$ e observações independentes; simetria composta (CS) - variâncias $\left(\sigma^{2}+\sigma_{1}\right)$ e covariâncias $\left(\sigma_{1}\right)$ homogêneas; autoregressiva de primeira ordem $(\mathrm{AR}(1))$ - variâncias homogêneas $\left(\sigma^{2}\right)$ e covariâncias reduzindo-se exponencialmente à medida que se aumenta o intervalo de tempo $\left(\sigma^{2} \rho, \sigma^{2} \rho^{2}, \sigma^{2} \rho^{3} \ldots\right.$; em que $\rho$, a correlação entre cortes, é o parâmetro autorregressivo assumido $|\mathrm{r}|<1$ em processo estacionário); Toeplitz (TOEP) variâncias homogêneas $\left(\sigma^{2}\right)$ e covariâncias desiguais $\left(s_{i,}\right.$; cortes $\left.i \neq i^{\prime}\right)$; simetria composta heterogênea $(\mathrm{CSH})$ - variâncias desiguais $\left(\sigma_{\mathrm{i}}^{2}\right)$ e covariâncias entre cortes dadas por $\sigma_{i} \sigma_{i}, \rho$; Huynh-Feldt $(\mathrm{HF})$ - variâncias desiguais $\left(\sigma_{i}^{2}\right)$ e covariâncias dadas por $1 / 2\left(\sigma_{i+}^{2} \sigma_{i}^{2}\right)-\lambda$ (em que $\lambda$ é a diferença entre a média das variâncias e a média das covariâncias); autoregressiva de primeira ordem heterogênea $(\mathrm{ARH}(1))$ - com variâncias desiguais $\left(\sigma_{i}^{2}\right)$ e covariâncias reduzindose exponencialmente, conforme: $\left.\sigma_{i} \sigma_{i}, \rho, \sigma_{i} \sigma_{i}, \rho^{2} \ldots\right)$; não estruturada (UN) - todas as variâncias $\mathrm{e}$ covariâncias desiguais. Maior detalhamento sobre estas e outras estruturas da matriz R são disponíveis em SAS INSTITUTE (2012).

Para avaliar a qualidade do ajustamento proporcionado pelos modelos sob diferentes estruturas da matriz $\Sigma$, em conformidade com RESENDE et al. (2006), foram utilizados o critério de informação de Akaike (AIC) e o teste da razão de verossimilhanças (LRT). O critério AIC é baseado na teoria de decisão e, para evitar excesso de parametrização, penaliza os modelos com número grande de parâmetros. Assim, é definido pela expressão: $\mathrm{AIC}=-2 \mathrm{I}+2 \mathrm{p}$, em que I é o logaritmo neperiano da função de verossimilhança do modelo, e "p" é o número de parâmetros da matriz de variâncias-covariâncias $(\Sigma)$. Segundo este critério, o modelo de matriz de variância-covariância a ser escolhido é aquele com o menor valor de AIC. Para compatibilizar a comparação dos valores de AIC de todos os modelos (com diferentes estruturas de $\Sigma$ ), o modelo univariado clássico foi ajustado sem o termo associado ao "erro a" (em nível de parcelas). Embora sem levar em conta a parcimônia do modelo, a estatística -2l, também denotada “-2 Reml" (relativo ao método de estimação de variâncias por "máxima verossimilhança restrita"), representa uma medida adicional da qualidade de ajustamento; em que menor valor implica melhor ajuste.

O teste LRT é utilizado para comparar dois modelos estimados por máxima verossimilhança, sendo um deles uma versão restrita do outro (modelos aninhados ou encaixados); isto é, 'modelo completo' versus 'modelo reduzido', com o primeiro tendo ' $r$ ' parâmetros adicionais. A estatística do teste avalia se os parâmetros adicionais melhoram significativamente o modelo. Assim, considerandose $L_{1}=-2$, para o modelo com menor número de parâmetros, e $\mathrm{L}_{2}=-2 \mathrm{l}$ para o modelo com maior número de parâmetros (com $r$ parâmetros extras), a hipótese em teste é a de que os dois modelos são equivalentes; isto é, os parâmetros extras não diferem de zero. Sob normalidade, a diferença entre $\mathrm{L}_{1}$ e $\mathrm{L}_{2}$ é assintoticamente distribuída como: $\mathrm{L}_{1}-\mathrm{L}_{2}$ $\sim \chi_{\text {[r] }}^{2}$ (qui-quadrado com $r$ graus de liberdade).

As análises foram realizadas no programa estatístico SAS (SAS INSTITUTE, 2012), via procedimento para ajuste de modelos lineares mistos (Proc Mixed).

\section{RESULTADOS E DISCUSSÃO}

Antes de comparar a qualidade de ajustamento associado às diferentes modelagens, é importante destacar certos resultados preliminares. Um deles refere-se à correlação entre cortes nos diferentes ensaios, que se manifestou com tendência positiva, embora de baixa magnitude ( $\mathrm{IC}^{95 \%}$ : $0,25 \pm 0,09)$. O segundo corte foi o que se mostrou mais correlacionado com os outros $(\mathrm{r}=0,32)$. Assim, não se confirmou a hipótese comumente aventada de falta de correlação entre as produções em cana-planta e nos demais cortes $(\mathrm{r}=0,28)$, o que ratifica a dependência natural entre cortes. Demonstra-se, portanto, que a suposição de independência não pode ser admitida como regra para sustentar um modelo de análise de variância clássica para esses ensaios (COSTA, 2003; FREITAS et al., 2008; QUINTAL, 2013). Tal constatação ainda foi corroborada pelos resultados significativos $(\mathrm{P}<0,01)$ do teste de esfericidade (MAUCHLY, 1940), aplicado a cada ensaio. Tudo isso justificou a investigação por alternativas de análise que acomodassem correlações dessa natureza.

Observando-se os resultados do ajuste dos modelos em cada ensaio, naquele conduzido 
em Jandaia, o modelo com matriz $\Sigma$ não estruturada (UN) foi o que apresentou melhor ajustamento aos dados (menores valores de -2 Reml e AIC); tanto assumindo os efeitos genotípicos como fixos ou aleatórios (Tabela 1). Esta superioridade foi confirmada pela significância $(\mathrm{P}<0,05)$ dos testes de razão de verossimilhanças, que compararam este modelo com os que imediatamente o sucederam nos valores de AIC (Tabela 2). A comparação estatística entre os demais pares sucessivos de modelos também ratifica a constatação de que a mudança na suposição dos efeitos genotípicos não afetou significativamente a sua ordem de classificação. Observa-se ainda que as estruturas PSC, VC e CS foram as mais inadequadas ao ajustamento dos dados deste ensaio, as quais não se diferenciaram $(\mathrm{P}>0,05)$, seja na suposição de genótipos fixos ou aleatórios. Logo, não se recomendaria o modelo univariado clássico (PSC) para a análise deste conjunto de dados.

No primeiro dos ensaios em Goiatuba (Série 95 de cruzamentos), a classificação dos modelos com diferentes estruturas de $\Sigma$ mostrou certa variação sob mudança na suposição dos efeitos genotípicos (Tabela 3). Isso, contudo, não afetou estatisticamente a eleição dos melhores e piores modelos, em ambos os casos. Tanto sob genótipos fixos, quanto sob genótipos aleatórios, as estruturas com os menores valores de AIC (melhor ajustamento aos dados) e que não diferiram estatisticamente entre si $(\mathrm{P}>0,05)$ foram $\mathrm{CSH}$ (simetria composta heterogênea) e UN (não estruturada). $\mathrm{O}$ bom ajustamento associado à estrutura $\mathrm{CSH}$ também foi observado por CECON et al. (2008). Já as piores estruturas (maiores valores de AIC), que também não diferiram entre si, embora de todas as demais, foram PSC, VC e AR(1).

Quanto às duas melhores estruturas, $\mathrm{CSH}$ e UN, vale destacar que, embora não se diferiram $(\mathrm{P}>0,05)$, a primeira delas é vantajosa, pois requer menor número de parâmetros para garantir a mesma qualidade de ajustamento. Entre aquelas de desempenho intermediário, houve considerável mudança na classificação. Por exemplo, enquanto, na condição de genótipos fixos, a estrutura HF ficou entre as três melhores; na de genótipos aleatórios, esta ficou entre as de desempenho intermediário (Tabela 3). O mesmo aconteceu com a estrutura TOEP, ao se inverter tais suposições. Isso demonstra que a especificação inadequada acerca da suposição do efeito genotípico também pode induzir à tomada de decisão errônea sobre a escolha da melhor estrutura da matriz de variâncias-covariâncias residuais. Por último, deve-se destacar o mau desempenho de ajustamento proporcionado pela estrutura PSC, que, tal como no ensaio anterior, ratifica a não recomendação do modelo univariado clássico. Isso corrobora as recomendações de que seja essencial modelar a estrutura de covariâncias na análise de dados longitudinais, para se garantir inferências válidas (QUINTAL, 2013).

No outro ensaio conduzido em Goiatuba (Série 96), as duas suposições para os efeitos genotípicos (fixos ou aleatórios) também praticamente não alteraram a identificação da melhor

Tabela 1 - Resultados do ajustamento de modelos com diferentes estruturas de matriz de variâncias-covariâncias residuais ( $\Sigma$ ), segundo o número de parâmetros (NP), valor máximo da função de verossimilhança (-2 Reml) e do critério de informação de Akaike (AIC), em análises de medidas repetidas de ensaio de competição de variedades de cana-de-açúcar, conduzido em Jandaia-GO (2005-2008), sob avaliação de três cortes e efeitos genotípicos assumidos como fixos ou aleatórios.

\begin{tabular}{|c|c|c|c|c|c|c|c|}
\hline Modelos & $\mathrm{NP}$ & -2 Reml & AIC & Modelos & $\mathrm{NP}$ & -2 Reml & AIC \\
\hline UN & 6 & 2248,601 & 2260,601 & UN & 8 & 2924,906 & 2940,906 \\
\hline ARH (1) & 4 & 2255,703 & 2263,703 & ARH(1) & 6 & 2933,739 & 2945,739 \\
\hline $\mathrm{CSH}$ & 4 & 2257,004 & 2265,004 & $\mathrm{CSH}$ & 6 & 2935,471 & 2947,471 \\
\hline $\mathrm{HF}$ & 4 & 2263,618 & 2271,618 & $\mathrm{HF}$ & 6 & 2941,500 & 2953,500 \\
\hline $\mathrm{AR}(1)$ & 2 & 2283,700 & 2287,700 & $\operatorname{AR}(1)$ & 4 & 2958,734 & 2966,734 \\
\hline TOEP & 3 & 2283,735 & 2289,735 & TOEP & 5 & 2958,489 & 2968,489 \\
\hline $\mathrm{CS}$ & 2 & 2286,397 & 2290,397 & $\mathrm{CS}$ & 4 & 2962,379 & 2970,379 \\
\hline $\mathrm{VC}$ & 1 & 2289,700 & 2291,709 & $\mathrm{VC}$ & 3 & 2965,200 & 2971,200 \\
\hline $\mathrm{PSC}^{1}$ & 1 & 2289,709 & 2291,709 & PSC & 3 & 2965,200 & 2971,200 \\
\hline
\end{tabular}

${ }^{1}$ Para obtenção de valores de AIC comparáveis, o modelo PSC (parcelas subdivididas), correspondente à estrutura de erros independentes, foi ajustado sem o termo relativo ao erro "a" (genótipos x blocos). 
Tabela 2 - Comparação de modelos com diferentes estruturas de matriz de variâncias-covariâncias residuais $(\Sigma)$, tomados dois a dois, segundo o teste da razão de verossimilhanças $\left(\chi^{2}\right)$, baseado em análises de medidas repetidas de experimento de competição de variedades de cana-de-açúcar, conduzido em Jandaia-GO (2005-2008), sob avaliação de três cortes e assumindo os efeitos genotípicos como fixos ou aleatórios.

\begin{tabular}{|c|c|c|c|c|c|c|c|}
\hline Estruturas & $\mathrm{DNP}^{1}$ & $\chi^{2}$ & p-valor & Estruturas & DNP & $\chi^{2}$ & p-valor \\
\hline UN-ARH(1) & 2 & 7,110 & 0,028581 & UN-ARH(1) & 2 & 8,790 & 0,012339 \\
\hline UN-CSH & 2 & 8,340 & 0,015452 & UN-CSH & 2 & 10,47 & 0,005327 \\
\hline UN-HF & 2 & 14,94 & 0,000570 & UN-HF & 2 & 16,58 & 0,000251 \\
\hline UN-AR(1) & 4 & 35,07 & $<0,00001$ & UN-AR(1) & 4 & 33,78 & 0,000001 \\
\hline UN-TOEP & 3 & 35,06 & $<0,00001$ & UN-TOEP & 3 & 33,61 & $<0,00001$ \\
\hline UN-CS & 4 & 37,44 & $<0,00001$ & UN-CS & 4 & 37,45 & $<0,00001$ \\
\hline UN-VC & 5 & 41,11 & $<0,00001$ & UN-VC & 5 & 40,29 & $<0,00001$ \\
\hline UN- PSC ${ }^{2}$ & 5 & 41,11 & $<0,00001$ & UN- PSC & 5 & 40,29 & $<0,00001$ \\
\hline ARH(1)-AR(1) & 2 & 27,66 & $<0,00001$ & ARH(1)-AR(1) & 2 & 25,00 & 0,000004 \\
\hline ARH(1)-TOEP & 1 & 27,94 & $<0,00001$ & ARH(1)-TOEP & 1 & 24,82 & $<0,00001$ \\
\hline $\mathrm{ARH}(1)-\mathrm{CS}$ & 2 & 30,67 & $<0,00001$ & ARH(1)-CS & 2 & 28,66 & $<0,00001$ \\
\hline ARH(1)- VC & 3 & 34,00 & $<0,00001$ & $\mathrm{ARH}(1)-\mathrm{VC}$ & 3 & 31,50 & $<0,00001$ \\
\hline ARH(1)-PSC & 3 & 34,00 & $<0,00001$ & ARH(1)- PSC & 3 & 31,05 & $<0,00001$ \\
\hline CSH-AR(1) & 2 & 26,73 & 0,000002 & CSH-AR(1) & 2 & 23,31 & 0,000009 \\
\hline CSH-TOEP & 1 & 26,72 & $<0,00001$ & CSH-TOEP & 1 & 23,14 & 0,000002 \\
\hline CSH-CS & 2 & 29,45 & $<0,00001$ & CSH-CS & 2 & 26,98 & $<0,00001$ \\
\hline $\mathrm{CSH}-\mathrm{VC}$ & 3 & 32,77 & $<0,00001$ & CSH-VC & 3 & 29,82 & 0,000002 \\
\hline CSH-PSC & 3 & 32,77 & $<0,00001$ & CSH-PSC & 3 & 29,82 & 0,000002 \\
\hline HF-AR(1) & 2 & 20,14 & 0,000042 & HF-AR(1) & 2 & 17,20 & 0,000184 \\
\hline HF-TOEP & 1 & 20,12 & 0,000007 & HF-TOEP & 1 & 17,03 & 0,000037 \\
\hline HF-CS & 2 & 22,85 & 0,000011 & HF-CS & 2 & 20,86 & 0,000030 \\
\hline HF-VC & 3 & 26,18 & 0,000009 & HF-VC & 3 & 23,70 & 0,000029 \\
\hline HF-PSC $^{2}$ & 3 & 26,18 & 0,000009 & HF- PSC & 3 & 23,70 & 0,000029 \\
\hline AR(1)-TOEP & 1 & 0,020 & 0,887537 & AR(1)-TOEP & 1 & 0,170 & 0,680112 \\
\hline $\mathrm{AR}(1)-\mathrm{VC}$ & 1 & 6,040 & 0,013985 & $\mathrm{AR}(1)-\mathrm{VC}$ & 1 & 6,510 & 0,010727 \\
\hline AR(1)-PSC & 1 & 6,040 & 0,013985 & AR(1)-PSC & 1 & 6,510 & 0,010727 \\
\hline TOEP-CS & 1 & 2,730 & 0,098479 & TOEP-CS & 1 & 3,840 & 0,050044 \\
\hline TOEP-VC & 2 & 6,060 & 0,048316 & TOEP-VC & 2 & 6,680 & 0,035437 \\
\hline TOEP-PSC & 2 & 6,060 & 0,048316 & TOEP-PSC & 2 & 6,680 & 0,035437 \\
\hline CS-VC & 1 & 3,330 & 0,068027 & CS-VC & 1 & 2,840 & 0,091944 \\
\hline CS-PSC & 1 & 3,330 & 0,068027 & CS- PSC & 1 & 2,840 & 0,091944 \\
\hline
\end{tabular}

${ }^{1}$ Diferença do número de parâmetros entre os dois modelos.

${ }^{2}$ Para obtenção de valores de AIC comparáveis, o modelo PSC (parcelas subdivididas), correspondente à estrutura de erros independentes, foi ajustado sem o termo relativo ao erro "a" (genótipos x blocos).

estrutura para a matriz $\Sigma$. Pela semelhança com o ensaio anterior, no mesmo local, estes resultados não estão aqui apresentados; são disponíveis em SILVA (2011). No caso de genótipos fixos, destacaramse as estruturas TOEP, CS, CSH e UN; porém, nenhuma destas superou estatisticamente, em nível de $5 \%$ de probabilidade, a matriz não estruturada (UN). FREITAS et al. (2005) também destacaram a qualidade de ajuste associada a esse tipo de matriz de covariâncias. Sob a suposição de genótipos aleatórios, outra vez destacaram-se TOEP, UN e CSH, as quais não diferiram entre si $(\mathrm{P}>0,05)$. Entre as piores estruturas, novamente estiveram as matrizes $\mathrm{VC}$ e PSC, que não se diferiram. Logo, o resultado reforça a inadequação das estruturas de erros independentes para o bom ajustamento dos dados.

Para o ensaio conduzido na localidade de Goianésia, outra vez a suposição associada aos efeitos dos genótipos (fixos ou aleatórios) praticamente não alterou a ordem de classificação das estruturas de $\Sigma$ (Tabela 4); e, novamente, destacou-se $(\mathrm{P}<0,01)$, com melhor qualidade de ajustamento, a matriz não estruturada (UN). Também se ratificou o pior desempenho das estruturas PSC e VC em relação 
Tabela 3 - Resultados do ajustamento de modelos com diferentes estruturas de matriz de variâncias-covariâncias residuais ( $\Sigma$ ), segundo o número de parâmetros (NP), valor máximo da função de verossimilhança (-2 Reml) e do critério de informação de Akaike (AIC), em análises de medidas repetidas de ensaio de competição de variedades de cana-de-açúcar, conduzido em Goiatuba-GO (2004-2008), sob avaliação de quatro cortes e efeitos genotípicos assumidos como fixos ou aleatórios.

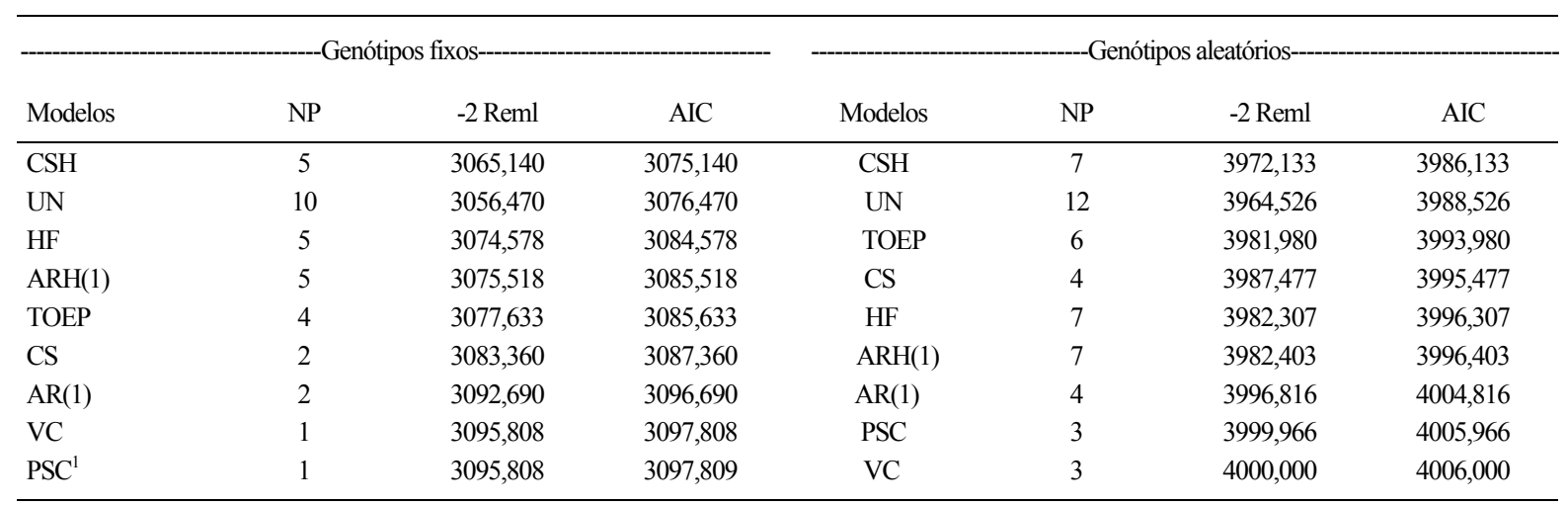

${ }^{1}$ Para obtenção de valores de AIC comparáveis, o modelo PSC (parcelas subdivididas), correspondente à estrutura de erros independentes, foi ajustado sem o termo relativo ao erro "a" (genótipos x blocos).

às demais. Ainda vale reportar que a suposição de genótipos fixos também não influenciou nas estimativas das médias genotípicas, mesmo sob as diferentes estruturas de matriz de variânciascovariâncias (SILVA, 2011). Este resultado corrobora a tese de que a suposição de tratamentos fixos é independente do tipo de estrutura de $\Sigma$, se os conjuntos de dados forem balanceados e ortogonais (DUARTE \& VENCOVSKY, 2001; CANDIDO, 2009).

\section{CONCLUSÃO}

A análise clássica sob erros independentes $\left(\Sigma=\mathrm{I} \sigma^{2}\right)$, denotada como modelo de parcelas subdivididas (PSC), oscilou entre as piores estruturas, ou, apenas, entre estruturas medianas quanto à qualidade de ajustamento dos dados. Logo, não se mostra adequada para a análise de ensaios varietais com medidas repetidas em cana-de-açúcar. Além disso, a melhor estrutura quanto à capacidade de o modelo ajustar-se aos dados observados varia entre ensaios, o que revela impossibilidade de se indicar previamente uma estrutura para a análise desse tipo de experimento. Por fim, a suposição relacionada aos efeitos genotípicos (fixos ou aleatórios), necessária na abordagem de modelos mistos, praticamente não altera a classificação das estruturas de matriz de variâncias-covariâncias residuais.

Tabela 4 - Resultados do ajustamento de modelos com diferentes estruturas de matriz de variâncias-covariâncias residuais ( $\Sigma$ ), segundo o número de parâmetros (NP), valor máximo da função de verossimilhança (-2 Reml) e do critério de informação de Akaike (AIC), em análises de medidas repetidas de ensaio de competição de variedades de cana-de-açúcar, conduzido em Goianésia-GO (2005-2009), sob avaliação de quatro cortes e efeitos genotípicos assumidos como fixos ou aleatórios.

\begin{tabular}{|c|c|c|c|c|c|c|c|}
\hline Modelos & NP & -2 Reml & $\mathrm{AIC}$ & Modelos & $\mathrm{NP}$ & -2 Reml & AIC \\
\hline UN & 10 & 1961,266 & 1981,266 & UN & 12 & 2804,886 & 2828,886 \\
\hline $\mathrm{AR}(1)$ & 2 & 1999,396 & 2003,396 & $\mathrm{AR}(1)$ & 4 & 2839,574 & 2847,574 \\
\hline TOEP & 4 & 1995,417 & 2003,417 & TOEP & 6 & 2837,276 & 2849,276 \\
\hline $\mathrm{HF}$ & 5 & 1993,888 & 2003,888 & ARH(1) & 7 & 2838,935 & 2852,935 \\
\hline ARH(1) & 5 & 1998,233 & 2008,233 & $\mathrm{HF}$ & 7 & 2839,959 & 2853,959 \\
\hline $\mathrm{CS}$ & 2 & 2011,828 & 2015,828 & $\mathrm{CS}$ & 4 & 2860,306 & 2868,306 \\
\hline $\mathrm{CSH}$ & 5 & 2010,601 & 2020,601 & $\mathrm{CSH}$ & 7 & 2855,911 & 2869,911 \\
\hline $\mathrm{PSC}^{1}$ & 1 & 2049,100 & 2051,100 & PSC & 3 & 2898,201 & 2902,201 \\
\hline $\mathrm{VC}$ & 1 & 2049,100 & 2051,100 & $\mathrm{VC}$ & 3 & 2898,201 & 2902,201 \\
\hline
\end{tabular}

${ }^{1}$ Para obtenção de valores de AIC comparáveis, o modelo PSC (parcelas subdivididas), correspondente à estrutura de erros independentes, foi ajustado sem o termo relativo ao erro "a" (genótipos x blocos).

Ciência Rural, v.45, n.6, jun, 2015. 


\section{AGRADECIMENTOS}

Ao Programa de Melhoramento Genético da Canade-Açúcar (PMGCA), Universidade Federal de Goiás (UFG), vinculado à Ridesa Brasil, pela seção dos dados e pela bolsa de estudos concedida ao primeiro autor, durante seu curso de mestrado no Programa de Pós-Graduação em Agronomia.

\section{REFERENCIAS}

CAMARINHA FILHO, J.A. Modelos lineares mistos: estruturas de matrizes de variâncias e covariâncias e seleção de modelo. 2002. 85f. Tese (Doutorado em Agronomia: Estatística e Experimentação Agronômica) - Escola Superior de Agricultura Luiz de Queiroz, Universidade de São Paulo, Piracicaba, SP.

CANDIDO, L.S. Modelos mistos na avaliação e ordenação de genótipos de cana-de-açúcar, com e sem efeitos de competição com parcelas vizinhas. 2009. 76f. Tese (Doutorado em Agronomia: Genética e Melhoramento de Plantas) - Faculdade de Ciências Agrárias e Veterinária Campus Jaboticabal, Universidade Estadual Paulista "Júlio de Mesquita Filho", Jaboticabal, SP.

CECON, P.R. et al. Análise de medidas repetidas na avaliação de clones de café 'Conilon'. Pesquisa Agropecuária Brasileira, v.43, p.1171-1176, 2008. Disponível em: $<$ http://seer.sct.embrapa br/index.php/pab/article/view/241/5497>. Acesso em: 17 out 2014. doi: 10.1590/S0100-204X2008000900011.

COSTA, S.C. Modelos lineares generalizados para dados longitudinais. 2003. 110f. Tese (Doutorado em Estatística e Experimentação Agronômica) - Escola Superior de Agricultura "Luiz de Queiroz", Universidade de São Paulo, SP.

DUARTE, J.B.; VENCOVSKY, R. Estimação e predição por modelo linear misto com ênfase na ordenação de médias de tratamentos genéticos. Scientia Agricola, v.58, p.109-117, 2001. Disponível em: <http://www.scielo.br/pdf/sa/v58n1/ a17v58n1.pdf>. Acesso em: 17 out. 2014. doi: 10.1590/S010390162001000100017.

FLORIANO, E.P. et al. Ajuste e seleção de modelos tradicionais para série temporal de dados de altura de árvores. Ciência
Florestal, v.16, n.2, p.177-199, 2006. Disponível em: <http:// coral.ufsm.br/cienciaflorestal/artigos/v16n2/A6V16N2.pdf>. Acesso em: 17 out. 2014

FREITAS, A.R. et al. Alternativas de análises em dados de medidas repetidas de bovinos de corte. Revista Brasileira de Zootecnica, v.34, p.2233-2244, 2005. Disponível em: <http://www.scielo.br/ pdf/rbz/v34n6s0/a10v3460.pdf $>$. Acesso em: 17 out. 2014. doi: $10.1590 / \mathrm{S} 1516-35982005000700010$

FREITAS, E.G. Análise de dados longitudinais em experimentos com cana-de-açúcar. 2007. 75f. Dissertação (Mestrado em Estatística e Experimentação agronômica) - Escola Superior de Agricultura Luiz de Queiroz, Piracicaba, SP.

FREITAS, E.G. et al. Modelo univariado aplicado a dados longitudinais de cana-de-açúcar. Revista Brasileira de Biometria, v.26, p.93-16, 2008. Disponível em: <http://jaguar.fcav.unesp.br/RME/fasciculos/ v26/v26 n2/A6 Edjane.pdf $>$. Acesso em: 17 out. 2014.

MAUCHLY, J.W. Significance test for a normal n-variate distribution. Annals of Mathematical Statistics, v.11, p.204-209, 1940.

QUINTAL, S.S.R. Melhoramento da goiabeira $P$. guajava via metologia de modelos mistos. 2013. 193f. Tese (Doutorado em Produção Vegetal) - Programa de PósGraduação em Produção Vegetal, Universidade Estadual do Norte Fluminense, RJ. Disponível em: <http://uenf.br/ Uenf/Downloads/PRODVEGETAL_3434_1379508554.pdf>. Acesso em: 17 out. 2014.

RESENDE, M.D.V. et al. Multivariate spatial statistical analysis of longitudinal data in perennial crops. Revista de Matemática e Estatística, v.24, p.147-169, 2006. Disponível em: <http://jaguar. fcav.unesp.br/RME/fasciculos/v24/v24_n1/A9_MDeon.pdf $>$. Acesso em: 17 out. 2014.

SILVA, E.N. Análise de medidas repetidas em ensaios varietais de cana-de-açúcar. 2011. 96f. Dissertação (Mestrado em Agronomia: Genética e Melhoramento de Plantas) - Programa de Pós-Graduação em Agronomia, Universidade Federal de Goiás, GO. Disponível em: < http://www.files.scire.net.br/atrio/ufg-ppga upl/THESIS/252/dissertao_emerson_noleto.pdf >. Acesso em: 17 out. 2014. 